barriers and facilitators to their proper dissemination and implementation; identify the strategies and actions for improvement that contribute to minimising the impact of the barriers that have been detected.

Methods We conducted a survey in order to assess resources, knowledge and attitudinal barriers of physicians working in Specialised Care towards the Clinical Practice Guidelines.

Results The questionnaire was completed by 209 SC physicians. The application of the recommendations in the CPGs is considered to be easy by $61.2 \%$ of participants, while $28.2 \%$ considered this procedure to be difficult. Among the reasons behind the difficulty were: the complicated nature of practical application, the lack of organisational, financial and infrastructurerelated resources, the variable nature of the patients, the lack of time, little evidence with low-quality recommendations, disagreement, a lack of interest and motivation and the lack of knowledge of the CPGs due to unsatisfactory dissemination.

Conclusion Informed by the results of the survey, leading health authorities are making an effort to develop specially designed interventions to implement clinical practice guidelines, including an easily accessible online database.

\section{P317 IMPLEMENTATION OF EVIDENCE BASED HEALTHCARE AND GUIDELINES IN CLINICAL PRACTICE}

${ }^{1} \mathrm{~K}$ Steinhausen, ${ }^{2} \mathrm{~S}$ Slordahl. ${ }^{1}$ Furtwangen University and European Science Foundation, Strasbourg, France; ${ }^{2}$ Norwegian University of Science and Technology and European Science Foundation, Trondheim, Norway

\section{0:1136/bmjqs-2013-002293.254}

Background Healthcare received by Europe's citizens should be based on the best scientific evidence and with involvement of patient and public. Greater emphasis on scientific evidence for a health intervention must be thoroughly analysed, health technology assessment (HTA) must become a cornerstone of healthcare. We have discussed these issues with different interdisciplinary groups and published two strategic papers in 2011 and 2012.

Objectives The aim is to present and discuss further possible implementation steps for improving implementation of evidence based healthcare and guidelines in clinical practice.

Methods In 2011 and 2012 workshops with interdisciplinary working groups (knowledge transfer, patient involvement and general practice) took place. Needs for actions and the relevant stakeholders were identified.

Results Needs for action: Establish a European Institute for Health Research where common issues in European healthcare research and policy can be debated and appropriate strategies formulated. Organise meetings between HTA/EBM leaders and policy-makers and health administrators on the European, national, regional and local level Establish at national level Healthcare Knowledge Centres for improved access to and transfer of unbiased information on patient-oriented research Set up research networks and ensure collaborative research between primary and secondary care Develop incentive systems for using and implementing evidence-based practice, guidelines and policy at medical care level through national European guidelines or even regulations and the relevant stakeholders.

Discussion It is now important to implement these needs. The involvement of different stakeholders from research, clinical practice, regulation, policy, patients and the public is urgently needed.
P319 MINDS PROJECT AS GUIDELINE CLEARINGHOUSE EVALUATION OF CLINICAL PRACTICE GUIDELINES DEVELOPED IN JAPAN

${ }^{1,2} \mathrm{~A}$ Okumura, ${ }^{1,3} \mathrm{M}$ Yoshida, ${ }^{1,4} \mathrm{~K}$ Kiyohara, ${ }^{1} \mathrm{~N}$ Takahashi, ${ }^{1,5} \mathrm{Y}$ Hatakeyama, ${ }^{1,6} \mathrm{~N}$ Htun, ${ }^{1,4} \mathrm{Y}$ Sato, ${ }^{1,4} \mathrm{~N}$ Kojimahara, MINDS Group ${ }^{1}, 1,4 \mathrm{~N}$ Yamaguch. ${ }^{1} \mathrm{MINDS}$ Center, Japan Council for Quality Health Care, Tokyo, Japan; ${ }^{2}$ Department of Social Medicine, The University of Tokyo, Tokyo, Japan; ${ }^{3}$ Department of Hemodialysis and Surgery, Chemotherapy Research Institute Inter, Tokyo, Japan; ${ }^{4}$ Japan, Department of Public Health , Tokyo, Japan; ${ }^{5}$ Women's Medical University, Tokyo, Department of Advanced Social and International Studies, Graduate School of A, Tokyo, Japan; ${ }^{6}$ Department of Molecular Epidemiology, Tokyo Medical and Dental University, Tokyo, Japan

\section{0:1136/bmjqs-2013-002293.255}

Background MINDS (Medical Information Network Distribution Service) is a consignment project for MHLW (Ministry of Health, Labour and Welfare) managed by Japan Council for Quality Health Care. MINDS has been disseminating evidencebased clinical practice guidelines (CPG) as guideline clearinghouse in Japan.

Objectives To assess the quality of evidence-based CPG developed in Japan.

Methods We searched Japanese CPG using 10 major databases from January 2007 to January 2013. After two-stage screening process with exclusion criteria, identified CPG were evaluated by 4 reviewers of the CPG evaluation group using the AGREEII (Appraisal of Guidelines for Research \& Evaluation II) Instrument.

Results A total of 1763 literatures were identified by the searching process. After screening process, 168 guidelines were evaluated by the AGREEII instrument from September 2011 to January 2013. The scores mean (SD) of each AGREEII domain were as follows: Scope and Purpose, 64.1 (19.2); Stakeholder Involvement, 46.0 (18.2); Rigour of Development, 39.8 (24.6); Clarity of Presentation, 58.8 (21.3); Applicability, 42.7 (16.3); Editorial Independence, 29.9 (31.4) and Overall assessment, 50.4 (21.1).

Discussion Among the AGREEII domains, Editorial Independence and Rigour of Development are important factors to improve the quality of Japanese CPG.

Implications for guideline developers/users It is necessary to cooperate with guideline development group in order to utilise the guidelines evaluation result for improving the guideline development process. MINDS is preparing to hold workshops 2013 focused on guideline methodology for guideline developers.

\section{P321 DISSEMINATION OF THE CLINICAL PRACTICE GUIDELINES DEVELOPMENT METHODOLOGY BASED ON BODY OF EVIDENCE IN JAPAN DEVELOPMENT OF EDUCATIONAL PACKAGE FOR CLINICAL PRACTICE GUIDELINES AND WORKSHOP PROGRAM}

${ }^{1,2} \mathrm{M}$ Yoshida, ${ }^{1,3} \mathrm{Y}$ Hatakeyama , ${ }^{1,4} \mathrm{~A}$ Okumura, ${ }^{1} \mathrm{~N}$ Takahashi, ${ }^{1,5} \mathrm{~N}$ Kojimahara, ${ }^{1,5} \mathrm{~K}$ Kiyohara, ${ }^{1,5} \mathrm{Y}$ Sato, ${ }^{1,6} \mathrm{~N}$ Htun, ${ }^{1,5} \mathrm{~N}$ Yamaguchi. ${ }^{1} \mathrm{MINDS}$ (Medical Information Network Distribution Service) Center, EBM Guidelines, Tokyo, Japan; ${ }^{2}$ Department of Hemodialysis and Surgery, Chemotherapy Research Institute, Inter, Ichikawa, Japan; ${ }^{3}$ Department of Advanced Social and International Studies, Graduate School of A, Tokyo, Japan; ${ }^{4}$ Department of Social Medicine, The University of Tokyo, Tokyo, Japan; ${ }^{5}$ Department of Public Health Tokyo Women Medical University, Tokyo Japan; ${ }^{6}$ Department of Molecular Epidemiology, Tokyo Medical and Dental University, Tokyo, Japan

10:1136/bmjqs-2013-002293.256 
Objective The dissemination and active support for clinical practice guidelines development method based on the principle of EBM, is one of the main pillars of MINDS (Medical Information Network Distribution Service) consignment project for EBM promotion implemented by Ministry of Health, Labour and Welfare Japan. In 2007, with the publication of MINDS guidance to develop Clinical practice guidelines 2007 by Fukui et al., a protocol to develop clinical practice guidelines was introduced as the most valid manual at that time in Japan. However, advanced improvements towards the global standards in the CPG development methodology have been established within these 5 years after previous publication, such as GRADE system, AGREE II, IOM etc. Accordingly, the Workshop for Clinical Practice Guidelines development methodology is planned to introduce the latest advanced methodology for medical and healthcare professionals who engaged in the clinical practice guidelines development. 2. Contents This workshop comprises the lectures and group practices, assuming AGREE II as a standard for the educational package development and, using GRADE system for quality of evidence and strength of recommendation, which promote the CPG development methods for disease management. We hope to show the 'PROGRAMME' for the progress of the workshop at G-I-N2013. 3. Target Audience For all medical and healthcare professionals who have interest in and for those who engage in clinical practice guidelines development in Japan. 4. Workshop Outline Educational workshops will be held four times within a year 2013 in Tokyo Japan. The first workshop date: 2013-03-20 (Wed) 10:00 17:00

\section{P326 IMPLEMENTING AN AUDIT AND FEEDBACK INTERVENTION TO REDUCE ANTIBIOTIC PRESCRIBING IN GENERAL DENTAL PRACTICE}

${ }^{1} \mathrm{~L}$ Young, ${ }^{1} \mathrm{D}$ Stirling, ${ }^{2} \mathrm{P}$ Elouafkaoui, ${ }^{1} \mathrm{~S}$ Rutherford, ${ }^{3} \mathrm{C}$ Ramsay, ${ }^{2,1} \mathrm{~J}$ Clarkson. ${ }^{1} T$ RiaDS, Research Methodology Group, NHS Education for Scotland, Dundee, UK; ${ }^{2}$ University of Dundee, Dundee, UK; ${ }^{3}$ University of Aberdeen, Aberdeen, UK

\section{0:1136/bmjgs-2013-002293.257}

Background In Scotland, guidance for prescribing in dentistry was published in 2008 in response to concerns about over-prescribing of antibiotics. The guidance recommends that antibiotic prescribing must be kept to a minimum. However, evidence from routinely collected data demonstrates that dental antibiotic prescribing is steadily increasing, now accounting for $9 \%$ of primary care antibiotic prescriptions.

Objectives To compare the effectiveness of different audit and feedback $(\mathrm{A} \& \mathrm{~F})$ strategies for the implementation of recommendations on dental antibiotic prescribing.

Methods The study is an 18 month, three-arm randomised controlled trial. All dentists in Scotland are being randomised to either: 1) access to a pre-approved national audit; 2) access to the audit plus individualised feedback; 3) access to the audit, individualised feedback and a persuasive message. The primary outcome is the number of antibiotic prescriptions per 100 patients. A process evaluation will be conducted to elucidate the mechanisms by which $A \& \mathrm{~F}$ influences practice. The trial was developed in collaboration with system-level stakeholders from policy, education and service providers.

Results Development of all interventions is complete and the trial will begin in March 2013. Processes are being developed to enable integration of the most effective intervention into national systems intended to reduce antibiotic prescribing.
Discussion Collaboration with system-level stakeholders has helped ensure that the trial addresses national priorities and has engendered system-level action for national implementation of the most effective intervention.

Implications for Guideline Developers/Users Generating robust experimental evidence on an intervention's effectiveness and collaboration with system-level stakeholders can increase the likelihood of its adoption as a policy initiative.

\section{P327 OVERVIEW OF CLINICAL PRACTICE GUIDELINES IN JAPAN - FROM THE POINT OF VIEW OF PATIENT- INVOLVEMENT}

H Suzuki. The International Medical Information Center, Tokyo, Japan

\section{0:1136/bmjqs-2013-002293.258}

Background In Japan, the official movement to develop Clinical Practice Guidelines (CPGs) began in 1999 with the financial support of the Ministry of Health, Labour and Welfare -MHLW). Since then, CPGs in various fields have been developed and development methods using the principles of evidence-based medicine are becoming popular.

Objectives The First objective of the study is to clarify how many CPGs developed and published in Japan in 2013. The second is to make clear if patient-involvement is popular or not.

Methods I have searched the existing CPGs comprehensively. Out of the 600 searched CPGs, well-formulated ones were selected if they met the following criteria: defining clinical questions to be addressed, reviewing evidence, determinig grade of recommendation, and becoming open to the public. I have checked public involvement in developing.

Results Nevertheless 120 CPGs are selected, the number of CPGs, containing patient/carer member in developing process, is only 6 . And the number of patient-version CPGs is only 29 .

Discussion A reason why the patient-involvement doesn't become popular, came from MHLW decision, stopping the financial support in 2004. This decision resulted in the view that individual professional societies were required to develop CPGs applicable to those topics related to their societies. Accordingly, a given professional society, which should always be involved in the CPG development group as one of the main stakeholders, was the sole party involved in the development of CPGs.

\section{P329 DEVELOPING A STRATEGY TO ASSESS THE REPORTING OF THE UPDATING PROCESS IN CLINICAL PRACTICE GUIDELINE: A DRAFT CHECKLIST}

1,2R Vernooij, 1,2A Sanabria, 1,2 L Martínez García, ${ }^{3} \mathrm{~J}$ Makarski, ${ }^{3} \mathrm{M}$ Brouwers, ${ }^{1,2} \mathrm{P}$ AlonsoCoello. 'Iberoamerican Cochrane Centre, Barcelona, Spain; ${ }^{2}$ Institute of Biomedical Research (IIB Sant Pau), Barcelona, Spain; ${ }^{3}$ Department of Oncology, McMaster University, Hamilton, Canada

\section{0:1136/bmjqs-2013-002293.259}

Background Scientific knowledge is in constant change and, therefore, clinical practice guidelines (CPGs) require a frequent reassessment. However, the best methodology for updating CPGs is not known and methods are poorly reported in CPGs. A framework to evaluate the quality of reporting the updating process in CPGs and to provide guidance for minimum thresholds for an updating strategy is needed.

Objective To develop a CPG update reporting checklist. 